\title{
Deutsch als "leicht zu erlernende Fremdsprache" 1
}

\author{
Gunde Kurtz (Kaiserslauten)
}

\begin{abstract}
The goal of this paper is to encourage the search for accessible language features and teaching and learning facilitators which can ease the way into German as a Foreign Language (DaF).

The paper begins with a terminological discussion, followed by a first model of the most important factors concerning the initial entry into FL territory. After a short review on previous work in this area, I consider methods for utilising knowledge of accessible language features and teaching and learning facilitators.

Finally, suspected facilitating features - which have come to light through research as well as classroom observations - are exemplified. These, however, are to be understood as working hypotheses and thus still 'under construction'.

Through this paper, I would like to invite other researchers to consider a new perspective on DaF with the intention of changing the status quo:

"Applied linguistics is not simply a matter of matching up findings about language with preexisting problems but of using findings to explore how the perception of problems might be changed. It may be that when problems are reformulated from a different point of view they become more amenable to solution. This changed perception may then, in turn, have implications for linguistics." (Cook : 2003: 10)
\end{abstract}

My goal will have been achieved when the focus of this paper becomes a motivation for improving DaF teaching ("problems [...] become more amenable to solution") - and, should it lead to "implications for linguistics", so much the better.

Ziel dieses Beitrages ist, eine umfassende Suche nach leichten Stellen und Erleichterungen beim Zugang zum Deutschen als fremder Sprache anzuregen.

\footnotetext{
${ }^{1} \mathrm{Neu}$ ist nicht das, was hier zusammengetragen ist (das meiste ist längst in der Literatur vorfindbar), sondern die Fokussierung auf die Frage nach der Leichtigkeit des Zugangs zu einer Sprache und die Sichtweise auf das

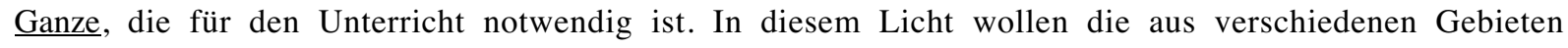
stammenden Einzelteile betrachtet werden. Denn das Grundproblem zwischen Theorie und Unterrichtspraxis ist, dass feinverzweigte Spezialgebiete, denen Forschungsgruppen viele Jahre widmen, auch auf viele Spezialisten verteilt sind. Andererseits soll nun all dies den Fremdsprachenunterricht verbessern helfen. Das heißt aber: Es muss ihn erreichen. Und da die Lehrenden die Ergebnisse aus allen Bezugswissenschaften unmöglich rezipieren können (wg. Masse, Fachsprachen und -"philosophien"), fehlt ein Zwischenglied - bis heute. Jedes solche Zwischenstück kann den strengen Ansprüchen der Spezialisten natürlich nicht mehr genügen, sonst wird es kaum seinen Weg in die Praxis finden. Was also hier auf den ersten Blick wie ein Sammelsurium aus zig disparaten Stücken aussieht, kann aus Sicht der Praxis durchaus ein sinnvolles Ganzes darstellen.
} 
Nach Begriffsklärung und einem ersten Vorschlag zu den für die Zugänglichkeit einer Sprache ausschlaggebenden Faktoren (1) sowie einem kurzen Überblick über Fundstücke zum Thema (2) steht die Rolle der praktischen Umsetzung von Erkenntnissen als Hauptinstrument für den Bereich der Erleichterungen und die Nutzung leichter Stellen im Mittelpunkt (3). Dabei werden einige Kandidaten benannt, die sowohl aus vorliegenden Forschungsergebnissen als auch aus Beobachtungen in Alltag und Fremdsprachenunterricht (FSU) gewonnen wurden. Die so gewonnenen Einsichten sind als Arbeitshypothesen und somit als im Wandel begriffen zu verstehen.

Ich möchte mit diesem Beitrag einladen zu einer neuen Perspektive auf das Deutsche als Fremdsprache, und zwar mit der Absicht etwas zu verändern:

"Applied linguistics is not simply a matter of matching up findings about language with preexisting problems but of using findings to explore how the perception of problems might be changed. It may be that when problems are reformulated from a different point of view they become more amenable to solution. This changed perception may then, in turn, have implications for linguistics." (Cook : 2003: 10)

Mein Ziel ist erreicht, wenn sich vorliegende Fragestellung als Schlüssel zu verbesserter DaFUnterrichtspraxis ("problems [...] become more amenable to solution") erweist. Falls sie sogar "implications for linguistics" zeitigt, umso besser.

Im Folgenden geht es um die Fragen, ob, wo und wie Deutsch als Fremdsprache leicht zu erlernen ist, leicht zugänglich ist oder Lernern erleichtert werden kann. Dazu sollen Maßstäbe und Bedingungen für Leichtigkeit und Zugänglichkeit gefunden werden sowie Ansätze zur Nutzung solchen Wissens für effektivere Sprachlernprozesse.

\section{Was die Titel-Begriffe hier bedeuten:}

- Deutsch: Hier sind zwei Ebenen zu unterscheiden, zum einen die deutsche Sprache von außen - auf ihre Zugänglichkeit für neue Lerner - betrachtet. Zum anderen lernersprachliches Deutsch: Ziel ist die Fähigkeit, erfolgreich an der Kommunikation im Alltag (Umgangssprache/einfache Variante) teilzunehmen, ggf. später weit ausgebaute Kenntnisse in "Wort und Schrift" zu erlangen.

- Fremdsprache: Im jugendlichen oder erwachsenen Alter erlernt - hier ist die Variante im Land der Zielsprache von Interesse. Der Unterschied zur Zweitsprache ist weniger wichtig, denn auch wenn DaZ gelernt wird, stellen sich die gleichen Fragen der Zugänglichkeit.

- Außerdem geht es hier besonders um frühe Erwerbsstadien und eine unterrichtsbezogene Erörterung der Problematik. 
- Lernen: Gezielte Beschäftigung mit einer Sprache mit der Absicht, sie zu benutzen, unter Einsatz von Hilfsmitteln oberhalb eines zweisprachigen Wörterbuches. Es kann sich z.B. um einen Sprachkurs mit Lehrkraft, Online-Lernen, Selbststudium oder den systematischen Einsatz von Immersionsstrategien handeln sowie um alle möglichen Mischformen.

- Leicht: Was bedeutet leicht im Hinblick auf eine (Fremd)Sprache? Diese Frage liegt der Suche nach leichter Zugänglichkeit zugrunde und wird uns hier weiter beschäftigen. Gibt es Maßstäbe für Leichtigkeit? Ist das Gegenteil schwer / komplex / fremd oder eher unfasslich / opak / gestaltlos?

Beginnen wir mit einem Gedankenexperiment und nehmen an, es existiert ein funktionstüchtiges Messinstrument zur Feststellung der Schwierigkeitsgrade von Sprachen, das es erlaubt, die äußerst komplexen Phänomene zu erfassen, zu bündeln und auf einer einfachen Skala abzubilden. Nehmen wir weiter an, dass jede Sprache in der Summe ihrer Schwierigkeiten die gleiche Zahl auf der Skala erreicht, und nennen wir die Zahl 100. Nun können wir die 100 Schwierigkeitseinheiten auf allgemeine Kategorien wie Phonetik, Graphie (ggf.), Lexik, Morphologie, Syntax, Semantik und Pragmatik und deren Unterkategorien verteilen. ${ }^{2}$ Wenn ein Gebiet sehr hohen Anteil an der 100 hat (also sehr schwer ist), bedeutet das, dass für andere Gebiete weniger Einheiten bleiben, dass sie also relativ leicht sein müssen. Dann wäre die Frage nach leichteren oder schwereren, nach leichter oder schwerer zu erlernenden Sprachen weitgehend überflüssig. Es wäre eher zu fragen, wie die Bereiche mit den kleineren Zahlen nutzbar gemacht werden können, um den Lernern einen effizienten Einstieg zu ermöglichen.

Dagegen stehen Annahmen der Evolutionstheorien, die (grob vereinfacht gesagt) Entstehungs- und Zerfallsprozesse im Leben von Sprachen erforschen. Das hieße also, dass Sprachen ihre Komplexität nicht nur auf-, sondern auch abbauen ${ }^{3}-$ z.B. durch Gebrauch in stark wachsenden Sprachgemeinschaften und durch natürliche Verfallsprozesse komplexer dynamischer Systeme. Geringere Komplexität wiederum sollte leichtere Zugänglichkeit bedeuten, mithin könnte eine bestimmte Sprache zu einer bestimmten Zeit eine leicht zu erlernende Fremdsprache sein, wie derzeit von vielen fürs Englische angenommen und z.B. von Franceschini/Klein (2003) unterstützt eben nicht unbedingt durch höhere Komplexität innerhalb des Sprachsystems erkauft werden müssen. Könnte es aber nicht auch so sein, dass die Vereinfachung des Sprachsystems durch höhere Komplexität in extralinguistischen Bereichen doch "bezahlt" werden muss - etwa durch Verkomplizierung in Aufbau, Handhabung und Erhalt von Identität und sozialen Netzen?

\footnotetext{
${ }^{2}$ Die Wahl der Kategorien spielt für das Bild des Messinstruments zunächst keine Rolle, natürlich könnte sie auch anders aussehen, z.B. könnte Semantik in die anderen Bereiche integriert sein oder es könnten (sprachabhängig) Gebiete wie Morphosyntax erstrangig relevant werden usw..

${ }^{3}$ Oder falls nicht, dass sie zum Untergang mangels Funktionalität (dazu zählt auch Prestige!) verurteilt sind.
} 
Die Suchrichtung, die aus der Annahme objektiv leicht erlernbarer Sprachen hervorginge, wäre die Etablierung von Kriterien, mit welchen sich die Leichtigkeit messen ließe. Ob und wenn ja wie solches Wissen allerdings den Fremdspracherwerb förderte? Möglicherweise dadurch, dass man "schwere" und "leichte" Bereiche sicherer orten und dadurch bei der Erstellung von Lehr-Lernplänen und -materialien gezielter berücksichtigen könnte, etwa in der Form: Elemente aus der Kategorie "leicht" werden immer mitgelernt, aus "schwer" wird (v.a. in frühen Lernphasen) nur das fokussiert, was auch das Kriterium "wichtig" erfüllt (s.u. Vorschlag Rug).

Zurück zum Beispiel der gleichen Gesamtschwierigkeit ${ }^{4}$ : Einige Evidenz spricht dafür, dass diese Annahme zumindest für die L1 zutreffen könnte. So ist z.B. erforscht, dass markante Phasen im Erstspracherwerb (ESE) sprachunabhängig im jeweils gleichen Alter abgeschlossen werden. Auch liegt die Idee einer Gesamtschwierigkeit nahe, wenn man Sprachen mit extrem differenzierten phonetischen Systemen aber z.B. ,ohne' Kasus, Numerus, Genus, Flexion oder variable Wortstellung betrachtet, oder Sprachen, die zwar in Morphologie und Syntax mit wenig Inventar auskommen und auch phonetisch nicht allzu komplex sind, aber intransparente (vielschichtige, feinverzweigte, seltene ...) semantische Differenzierungen in Lexik und Phraseologien nutzen usw..

Welche Suchrichtung würde aus der Annahme der "gleichen Summe" resultieren? Den ESE könnte man näher auf diejenigen Teile der Sprache untersuchen, die in unterschiedlichen Sprachen eben doch Abweichungen im Erwerbszeitplan zeigen, wobei im Vergleich auffällig frühe Einheiten von besonderem Interesse wären. Z.B. könnte die Tatsache, dass Morphologie beim ESE oft spät, im Türkischen aber vergleichsweise früh erworben wird, für den ZSE der jeweiligen Sprachen ebenfalls von Bedeutung sein.

Den muttersprachlichen Gebrauch erwachsener Sprecher könnte man auf solche Bereiche untersuchen, die von allen mit höchster Wahrscheinlichkeit sicher beherrscht werden (also gerade nicht die Bereiche, die selbst viele L1-Sprecher vor Probleme stellen). Diese Bereiche sollten für die Zieldefinition von Sprachunterricht und Lehr-Lernmaterialien gerade für weniger weit Fortgeschrittene eine zentrale Rolle spielen. ${ }^{5}$

Derartige Erkenntnisse aus ESE-Forschung und Erforschung des L1-Gebrauchs ergäben Hinweise auf Stellen, die Lernern als offene Türen zur neuen Sprache angeboten werden sollten. Da es hier aber um die Frage des Fremdspracherwerbs geht, müsste weiter differenziert werden. Denn die Eigenheiten einer Sprache sind dabei nur ein Faktor unter anderen, die einen Lerner erst zusammengenommen auf mehr oder weniger leichten Zugang festlegen, einen individuellen Lerner mit seiner Sprachbiographie, Lebenssituation und Motivation und seinen Lernmöglichkeiten. Überlegt man, welche Faktoren die Zugänglichkeit

\footnotetext{
4 Extreme Vertreter dieser These sind Bauer/Trudgill, die in Language Myths (1998) die Gleichwertigkeit aller Sprachen postulieren.

5 Dabei kann man durchaus eine einfache Variante der Umgangssprache annehmen und muss nicht auf Substandard bzw. regionale Varietäten zurückgreifen, wie das Schäfer wiederholt vorgeschlagen hat, zuletzt in Schäfer (2002). Denn auch wenn sein Vorschlag unter linguistischen Gesichtspunkten interessant sein mag, dürfte er bei Sprachlehrern - zumindest außerhalb des Ruhrgebietes - kaum auf Interesse stoßen.
} 
einer Sprache für ihre Lerner bestimmen, so kommt man zu einer Liste, die ungefähr so aussehen könnte: ${ }^{6}$ (Fett erscheint das, was von Seiten der Linguistik am leichtesten direkt zu beeinflussen und dann im FSU nutzbar ist, kursiv erscheint das, was von Seiten der Kurs- und Materialmacher außerdem nutzbar ist - und das ist fast alles, wenn man von der Einschränkung absieht, dass unter Punkt 4. nur das nutzbar wird, was im Hinblick auf den Lerner als positiv zu bewerten ist):

Faktoren, die die Zugänglichkeit einer Sprache für ihre Lerner bestimmen:

1. Eigenschaften der Sprache

1.1 Eigenschaften der Sprache als System Hierzu gehören Fragen nach mehr oder weniger komplexen Bereichen der Sprache, bzw. nach universalgrammatisch mehr oder weniger prototypisch / erwartbar ausgeformten Bereichen.

1.2 Eigenschaften der Sprache relativ zur Sprachbiographie des Lerners Hier müssen die Fragen aus 1.1 im Hinblick auf eine bestimmte linguistische Ausgangssituation erweitert bzw. neu ausgerichtet erörtert werden.

1.3 Eigenschaften der Sprachbeschreibung Hierhin gehört die Frage, ob eine Sprache für Arbeit an den unter 1.1, 1.2 und 3.2 genannten Aufgaben zweckmäßig beschrieben ist.

2. Eigenschaften des Lerners

2.1 Eigenschaften des Lerners hinsichtlich seiner Sprachlernbiographie

Hier sind diejenigen Faktoren einer Sprachlernbiographie ausfindig zu machen, die beim Einstieg in eine neue Sprache nützlich sind. Dabei steht nicht die unter 1.2 genannte linguistische Komponente der Sprachbiographie im Zentrum, sondern Lernerfahrungen speziell im Zusammenhang mit Sprache(n).

2.2 Eigenschaften des Lerners hinsichtlich seiner Situation \& Motivation

Hierzu gehören die Lebensumstände des Lerners einschließlich seiner Gründe, die Zielsprache zu lernen.

3 Eigenschaften des Lehr-Lernweges

3.1 Eigenschaften des Lehr-Lernweges hinsichtlich seiner raum-zeitlichen Intensität Hierzu gehört die Frage, ob immersiv gelernt wird (Kontaktmöglichkeiten) und wie viel Zeit für Kontakt und gezieltes Lernen der Sprache eingesetzt wird.

3.2 Eigenschaften der Gestaltung des Lehr-Lernweges in eine Sprache Hier geht es um die Eignung von Lehr-Lernmaterialien und -konzepten für den FSE, also um Fragen der Zielgruppenorientierung und der Effektivität.

4. Eigenschaften der Zielsprach-Gemeinschaft (sozio-kulturell)

4.1 Individueller Umgang mit Fremdem \& Fremden im Land im Hinblick auf den Lerner

\footnotetext{
${ }^{6}$ Diese Liste ist als Vorschlag zu verstehen und erhebt weder in ihrer Systematik noch in ihrer Länge Anspruch auf Vollständigkeit oder gar langfristige Gültigkeit.
} 
Hierzu gehören Fragen der Toleranz gegenüber "falsch gebrauchter Sprache" (insbes. "Akzent"), Zugänglichkeit/Integrationsfähigkeit der Gesellschaft, Reflexionsfähigkeit (insbes. im Hinblick auf Wertesysteme: "Ambiguitätstoleranz").

4.2 Offizieller Umgang mit Ausländern und Immigranten

Hierzu gehören neben Kontaktmöglichkeiten vor allem die Steuerung von Integration, Assimilation, Segregation.

Zu 1.: Es bedeutet natürlich einen großen Vorteil, den ersten Faktor (die Sprache selbst) genau zu kennen. Denn das Sprachsystem an sich ist zwar kaum beeinflussbar, wohl aber die Art seiner Vermittlung. Also ist dieser Faktor eine der beiden durch Sprachunterricht weitreichend beeinflussbaren Größen beim FSE, da die Lehrkomponente in Kursen und Materialien es erlaubt die zu unterrichtenden Sprachbereiche auszuwählen, zu gewichten und günstige Darstellungsformen einzusetzen. Lehrenden und Produzenten von LehrLernmaterialien praktikable Werkzeuge zur Identifizierung der jeweils günstigen Auswahl, Gewichtung und Darbietungsweise von Sprache an die Hand zu geben ist primär Aufgabe von Linguisten.

Zu 2.: Mit dem zweiten Bereich (besonders Sprachlernbiographien, Motivation) beschäftigt sich die Sprachlehrforschung DaF sehr intensiv. Allerdings gibt es nur wenige Ergebnisse, die so weit entwickelt bzw. konkretisiert sind, dass sie in der DaF-Praxis wirkungsvoll einsetzbar wären? .

Zu 3.: Lehr-Lernwege zu DaF werden ebenfalls intensiv erforscht und diskutiert, allerdings weniger unter dem Gesichtspunkt ihrer Effektivität im Hinblick auf die Teilnahme an alläglicher Kommunikation, sondern kurioserweise unter dem Blickwinkel, ob bestimmte Formen richtig gebildet werden oder bestimmte Tests oder Prüfungen bestanden, wobei meist der äußerst wichtige Zeitfaktor (Verhältnis von aufgewendeter Lernzeit zu Lernerfolg) gar nicht berücksichtigt wird.

Am "fatalsten" (im Sinne von unbeeinflussbar gegeben) erscheinen auf den ersten Blick die unter 4. genannten Bereiche. Aber auch hier können Fachleute in einem bestimmten Rahmen auf 4.2 einwirken und Lehrkräfte die unter 4.1 genannten Gegebenheiten auf Nützliches abklopfen und dies für den Lernprozess instrumentalisieren.

\footnotetext{
7 Manche Ergebnisse finden zwar Eingang in Lehrwerke, z.B. als Kapitel zu Sprachreflexion oder Lernstrategien, dies aber direkt $u$. unreflektiert. Ob auf diese Weise Lernerfolg erzielt wird, müsste allerdings überprüft werden. Nach meinem persönlichen Eindruck dienen diese Kapitel eher der Lehrerfortbildung denn als Verbesserung des Lernprozesses. Forschungsergebnisse in dieser Weise "umzusetzen" könnte erst die Vorstufe einer wirklichen Einarbeitung und Anwendung im Unterrichtsgeschehen sein, einer notwendig anderen Art, wie Sprache dort be- und verarbeitet wird. Die Hilflosigkeit der direkten Implementation erinnert an Versuche, Flirten in Kursen zu lehren, indem man betont, dass Charme (Begriff in Komponenten zerlegt und erklärt) sehr wichtig ist.
} 


\section{$2 \quad$ Fundstücke zum Thema}

Deutschlernenden werden Materialien mit dem Titel "Deutsch leicht" angeboten. Worauf das Versprechen gründet, bleibt unklar. Systematische Forschung dazu, wo und wie DaF leicht sein oder werden kann, wird m.W. derzeit nicht betrieben. In der Literatur konnte ich keinen Titel zur Frage der Leichtigkeit des Deutschen ausfindig machen, was zumindest darauf hinweist, dass solche Titel schwer zu finden sind. Zur verwandten Frage nach Schwierigkeiten, die Deutsch seinen Lernern bereitet, existiert dagegen einiges, wenn auch nur vereinzelt ${ }^{8}$.

In einem Tagungsband mit dem Titel "Wie schwer ist die deutsche Sprache wirklich?" (Wolff et al. : 2001), betrifft nur eine von fünf Sektionen diese Thematik. Das sind noch elf Beiträge auf über 150 Seiten. Aber nur einer dieser Beiträge, der von Carlo Serra Borneto (a), geht tiefer auf die Frage der Schwierigkeit ein. Die anderen gehören den folgenden Typen an: (b) Sie bemühen bekannte Stereotypen zur schweren, hässlichen Sprache und räumen Mark Twain u.ä. viel Platz ein, Erkenntnissen zum Thema kaum. Daher gehe ich hier nicht weiter darauf ein. (c) Sie beschränken sich auf fehler- und transfer-/ interferenzbezogene Aspekte von Schwierigkeit. Dabei bleibt der Begriff schwer / schwierig ungeklärt. (d) Nicht zu b/c gehörige Beiträge: Barkowski (2001 : 566 ff.) kommt zu dem Schluss, dass "Wahrnehmungslernen" (FSE im Alltag) bedeutungszentrierten Lexemerwerb erleichtert, während es andere Lernpotentiale nicht aktiviert, was also Aufgabe der Zweitsprachdidaktik wird. Hufeisen (2001) verweist auf die Potentiale, die in der Nutzung von Englisch als erster Fremdsprache für den DaF-Erwerb liegen. Fries (2001) bringt Licht in die Frage der Genera im Deutschen, ein traditionell als für Lerner besonders schwierig geltendes Gebiet, und zeigt systematische Zusammenhänge auf. Das entspricht dem Ansatz Erleichterung durch Fortbildung. Denn er erklärt DaF-Praktikern (Zielgruppe der Tagung) dieses Gebiet in differenzierter Weise. Das trägt sehr zur lehrerseitigen Klarheit über das Phänomen bei. Allerdings bleibt völlig offen, welche Konsequenzen sich daraus für die Lehr-Lernpraxis ergeben könnten. ${ }^{9}$

$\mathrm{Zu}$ (a): "'Schwer' ist, was für uns - oder in uns - ein Hindernis darstellt, und nicht nur etwas Objektives, Konkretes, außerhalb von uns Liegendes." (Serra Borneto : 2001 : 499)

Mit dieser Definition schließt Serra Borneto seine Betrachtungen zu intuitiven Vorstellungen vom Begriff schwer und zur Seltenheit fachmännischer Definitionen ab. Er zeigt, dass die Besprechung des Begriffs gewöhnlich in der Vermeidung einer Definition mündet und dass ältere Versuche entweder auf Kontrastives abzielen (wie bei Lado oder Kellerman) oder auf Subjektives (wie bei Corder). Serra Borneto macht geltend, dass Fehler alleine als Indizien für Schwierigkeit nicht ausreichen, da es auch andere Indikatoren wie Vermeidung, Verzögerung oder Missverständnis gibt. In seiner umfangreichen Studie zu Bereichen, die für fortgeschrittene Lerner des Deutschen schwer sein könnten (vgl. auch Serra Borneto 2000),

\footnotetext{
${ }^{8}$ Sollte die Frage nach Leichtigkeit auch dadurch verstellt sein, dass im Wortpaar leicht - schwer letzteres das Default-Wort ist? Ob daher zu fragen: Wie leicht ist das? ähnlich fern liegt wie etwa Wie jung bist du? oder Welche Schuhkleine hat das Kind??

${ }^{9}$ Diese Kluft wird vom Autor selbst in einem Schlusssatz betont, s.u.
} 
zeigt er unter anderem den wesentlichen Einfluss, den die Art der Fragestellung des Forschers auf die Nennungen der Probanden hat.

$\mathrm{Zu}$ (c): Die Beiträge dieser Gruppe zeigen, dass die Erkenntnisse kontrastiver oder fehlerorientierter Untersuchungen zwar wichtig sind, vielleicht aber zu anderen Schlussfolgerungen führen müssten, um auch wirksam zu werden. Hier seien zwei genannt: Demme (2001: 576) kommt zu der Einsicht, dass Ergebnisse ihrer Lernerbefragung zu schwierigen Gebieten weniger von der Muttersprache als vielmehr von der Art der Beschulung und von Eigendynamiken von Lernersprachen beeinflusst werden. Der Vorschlag von Rug (2001: 614), als wichtig und schwierig identifizierte Bereiche nach den drei Kriterien Relevanz, Kompliziertheit und Progressionszugehörigkeit weiter zu strukturieren, um ein Instrument für die Hand des Lehrers daraus zu bekommen, klingt plausibel. Dabei wird Kompliziertheit daran bemessen, ob der Bereich mit Regeln erfasst ist: Wenn ja, dann sei die Sache "einfach". Solches Vorgehen greift offensichtlich zu kurz ${ }^{10}$. Auch die angeführten Beispiele sind wenig überzeugend, zumal alle Zuordnungen ohne Begründung bleiben.

Zusammenfassend bleibt zu sagen: Schwierigkeit wird - intuitiv - noch zu sehr an Fehlerhäufigkeiten, Unregelmäßigkeiten oder vielschichtigen Paradigmen festgemacht. Dass hier Verbindungen bestehen, soll gar nicht von der Hand gewiesen werden. Jedoch ist zum Bereich "Fehler" zu bemerken, dass

- Daten zur Fehlerhäufigkeit bereits Grundannahmen zum Begriff des Fehlers und zu Fehlerbereichen unterliegen, welche für die Frage, wie weit Eigenschaften der Sprache Fehler provozieren, nicht unbedingt geeignet sein müssen.

- Zusammenhänge mit Unregelmäßigkeiten zumindest nicht zwingend sind, da Evidenz dafür vorliegt, dass auffällige Formen teilweise früh erlernt werden und leicht memorisierbar sind usw. (z.B. weil auch die Frequenz eine Rolle spielt).

- die Frage der Leichtigkeit nur sehr eingeschränkt über die der Schwierigkeiten zu beantworten ist, da die Zusammenhänge komplex sind.

- Daten zur Fehlerhäufigkeit wenig für die Frage der Leichtigkeit relevante Aufschlüsse liefern, wenn nicht zugleich auch die Fehlerursachen mit erhoben wurden. Denn schlechte Performanz kann durch andere Faktoren ausgelöst werden, etwa durch ungeeignete Lehr-Lernmethoden oder -inhalte, nicht zuletzt auch durch Stress.

$\mathrm{Zu}$ Zusammenhängen mit komplexen Paradigmen sei bemerkt, dass sich hier weniger die Frage stellt, auf welcher Lernstufe diese Schwierigkeiten bereiten, als die Frage, ob sie nicht später sogar eine Lernhilfe werden können. Zentral für vorliegende Fragestellung wäre hier, unter welchen Bedingungen komplexe Sprachbereiche den Zugang zu DaF nicht erschweren. Ausschlaggebend ist da mit hoher Wahrscheinlichkeit auch die Rolle, die diese Bereiche für

\footnotetext{
${ }^{10}$ Die Kenntnis systematischer Zusammenhänge führt allein längst nicht zur Anwendung im freien Sprechen oder Schreiben - diese schmerzliche Erfahrung teilen Lehrer und Lerner von Fremdsprachen. Die Frage ist vielmehr, ob und welche Konsequenzen sich aus derartigem Regelwissen für erfolgreiches Sprachenlernen ergeben.
} 
gelingende Kommunikation (v.a. in frühen Erwerbsphasen) spielen. Das erscheint plausibel, wenn man zwei Beispiele aus DaF näher betrachtet: Die Beherrschung sowohl der Deklination als auch die der Partikeln gilt als besonders schwierig. Erstere spielt für das Gelingen von Kommunikation eine eher marginale, letztere dagegen eine enorm wichtige Rolle. Für den Erwerb der Partikeln ist Aufenthalt und Kontakt in deutschsprachiger Umgebung erwartungsgemäß ausschlaggebend und nicht selten fast hinreichend, was für den Erwerb der Deklination ausgeschlossen ist: Hier geht es nur über disziplinierte, gezielte Übung. ${ }^{11}$

Zwar nicht der Frage nach DaF im engeren Sinne, aber der Frage nach Einfache(r) Sprache ist Heft 131 der Zeitschrift für Literaturwissenschaft und Linguistik gewidmet. Hier finden sich verschiedene Ansätze, Aspekten der Einfachheit von Sprachen auf die Spur zu kommen. In ihrem Vorwort gehen Franceschini/Klein (2003: 5ff.) zunächst dem intuitiven Konzept der Einfachheit nach:

"'einfach - schwierig' bezieht sich auf Handlungen, 'einfach - komplex' bezieht sich auf Strukturen. Beides steht oft im Zusammenhang, aber dieser Zusammenhang ist kein notwendiger." (5)

Sie bringen zur Frage nach dem Grad der Einfachheit einer Sprache oder eines Teilsystems davon auch die Frage nach der von einfachen und vereinfachten Strukturen leistbaren Funktionen (ihrer Funktionalität) ins Spiel.

Folgt man den Ausführungen Wunderlichs, "daß Grammatikalisierung gerichtet ist, ... und dass Morphologie zugunsten der Syntax abgebaut wird" (2003: 18), dann könnte man hieraus ein lernerspezifisches Kriterium für einfache Zugänglichkeit des Deutschen ableiten: Es sollte dann bei denjenigen Ausgangssprachen einfacher sein, für die Deutsch in der "natürlichen" Richtung (Abbau von Morphologie) liegt. "Große Sprachen" (ebd.: 20) zeichneten sich durch die "Interaktion von Wortstellung mit Topik und Fokus und die Tendenz zu Generalisierungen" (ebd.) aus. Da Wunderlich einen engen Zusammenhang mit der Sprecherpopulation und deren Kontakt mit Lernervarietäten sieht (ebd.: 16, 20), liegt es nahe, diese Eigenschaften als Folge einer Vereinfachung für größere Sprachgemeinschaften zu betrachten. Deutsch ist nach diesen Kriterien mit großen Schritten unterwegs zur "großen Sprache" und damit zur leichter (als früher) erlernbaren.

Klein (2003) stellt Möglichkeiten dar, welche Flexionsmorphologie als vollständig und ohne funktionale Verluste ersetzbar zeigen. Er beleuchtet vor allem Eigenheiten von sog. natürlich erworbenen Lernervarietäten und inwieweit diese funktional mehr oder weniger unbeschädigt ohne Flexionsmorphologie auskommen. Es ließe sich folgern, dass ein Unterricht, der dem natürlichen Erwerb folgt, den Zugang zur Fremdsprache leichter machte. Diese Frage ist zwar schon länger zugunsten der Feststellung beantwortet worden, dass Unterricht vor allem dazu dient, den FSE "unnatürlich" zu beschleunigen. Trotzdem scheinen mir viele Hinweise aus

\footnotetext{
11 Deklination wiederum gehört gerade zu denjenigen Gebieten, die auch von L1-Sprechern nicht mehr sicher beherrscht werden, vielleicht weil ihr "pragmatischer Nutzen" fast nur noch darin besteht, ein gewisses Bildungsniveau zu signalisieren.
} 
meiner Kenntnis der DaF-Praxis wertvoll und verwertbar. So z.B., dass reiche lexikalische Möglichkeiten zum Ausdruck von Tempus (und Aspekt) schon sehr früh hochfunktionale Kommunikation ermöglichen. Dies würde bedeuten, dass in frühen Erwerbsstadien v.a. das Unterrichten von solchem Funktionswortschatz hilfreich wäre - über den später die Funktion von Flexionselementen leichter erklärbar wird.

Dietrich (2003) ${ }^{12}$ unternimmt einen Versuch, den Begriff "einfache Sprache" (ebd.: 59) zu klären: Dieser kann sich auf strukturelle Eigenschaften der Sprache beziehen aber auch auf die Art, wie ein Sachverhalt sprachlich ausgedrückt wird, oder auf vereinfachte Register. Auf diesen letzten Punkt, Lernervarietäten, konzentriert sich der Beitrag. Dabei zeigt er anschaulich, dass "einfach" immer relativ zu bestimmten Zügen einer Sprache und stets komparativ, nie klassifikatorisch (ebd.: 60) verstanden werden sollte. Dietrich vertieft die Frage nach dem Verhältnis zwischen textlicher und kontextueller Information, die die Verständlichkeit von Äußerungen ermöglichen, und kommt letztlich zu dem Schluss, dass einfache Codes bald an ihre Grenzen stoßen, wenn der Kontext nicht weit trägt.

Für unsere Fragestellung bleibt hier nur festzustellen, dass ein Einstieg über erwartbare Themen und Kommunikationssituationen (also mit Hilfe tragfähigen Kontexts) immer eine Erleichterung bedeutet.

Ein neueres Forschungsgebiet, das ebenfalls Fundstücke für vorliegende Fragestellung liefert, stellt der Komplex L3-/Tertiärsprachenforschung, Mehrsprachigkeitsforschung, Interkomprehensionsforschung und Erforschung des multiplen Sprachenlernens dar. Im Rahmen dieser Ansätze beschäftigt sich das Groß-Projekt EuroCom mit der Frage des schnelleren und erleichterten Zugangs zu Fremdsprachen, wobei auch hier leicht/Erleichterung nicht definiert wird. Bei EuroCom steht ein Aspekt (Rezeption) einer regional beschränkten Gruppe von Sprachen (die drei großen Sprachfamilien Europas) unter einer bestimmten Voraussetzung (der Lerner hat Kenntnisse in einer nahe verwandten Sprache) zur Untersuchung. Es geht vor allem darum, die Sprachaufmerksamkeit auf bekannte verwandte Sprachen (sog. "Brückensprachen") zu lenken und sie beim Einstieg in die neu zu lernende Sprache zu nutzen. Für DaF betrifft das vor allem den Ansatz von Marx' Pilotstudie zum Hörverstehen (Marx : im Druck), die Nutzungsmöglichkeiten von Englisch als L2 für späteres DaF-Lernen (DaF nach Englisch) untersucht. Dies ist eine der aufzuspürenden und zu erforschenden Möglichkeiten, den Zugang zu DaF zu erleichtern, die, wenn sie weiter entwickelt wird, von Nutzen sein kann.

\section{Die Rolle der praktischen Umsetzung von Erkenntnissen als Hauptinstrument für Erleichterungen und die Nutzung leichter Stellen beim Zugang zu DaF}

Dass die Praxis des Fremdsprachenunterrichts mit den theoretischen und sogar den angewandten Bezugsdisziplinen nicht nur nicht Schritt hält, sondern auch über weite Strecken gar keine Verbindungen mehr bestehen, wird von beiden Seiten beklagt. Gründe dafür sind

\footnotetext{
12 Der Beitrag ist sehr eng an Klein/Dietrich 1986 angelehnt.
} 
sicherlich vielfältig. Ein wichtiger Grund dürfte die Vielzahl der Ergebnisse aus hochspezialisierten Bezugsdisziplinen sein, die dem integrierten Bedarf "aller Ergebnisse" für die Unterrichtssituation gegenüber steht. Ein scheinbar unmöglicher Anspruch. Trotzdem sehe ich dies nicht als unüberwindbare Mauer an. Wo könnte angesetzt werden?

Dazu noch einmal zu den Faktoren:

Wie müsste eine leicht lernbare Sprache aussehen? Welche sprachinternen Eigenschaften machen eine Sprache für erwachsene Lerner leicht zugänglich? Diese allgemeine Frage übersteigt bei weitem meine Möglichkeiten - sicher spielen hier Universalien, Optimalitätskriterien, Salienz, Kategorisierungsarten, "Einstiegs-Schwelle"13 (Mindesterwerbsstand, ab dem in dieser Sprache erfolgreich kommuniziert werden kann) u.a. eine wichtige Rolle.

Wir haben allen unseren Kursteilnehmern nach ihrem Einstiegskurs ${ }^{14}$ die Frage gestellt, was sie am Deutschen leicht finden. Unter vielen Antworten waren zwei fast immer dabei, völlig unabhängig von Sprachbiographie und Lernertyp: Dass der Wortschatz einfach (transparent, systematisch) und damit leicht lernbar sei und dass die Phonem-Graphem-Beziehungen schnell nachvollziehbar seien ${ }^{15}$. Weitere häufige Nennungen betreffen Aussprache, Intonation und die Tatsache, dass man auf Deutsch schon mit geringen Mitteln "alles" sagen kann. Fast alle betonen auch die Geduld der Einheimischen mit Anfängern, aber damit sind wir schon beim nächsten Punkt.

Was macht eine Fremdsprache leicht erlernbar? Unter welchen Bedingungen fällt uns eine neue Sprache leicht? Fassen wir zusammen, was Fundstücke (auch hier nicht eigens genannte) und Alltagserfahrung nahe legen:

1. Eine große Hilfe beim FSE ist die Motivation, die Lust auf die neue Sprache. Die ist gegeben, wenn man irgendetwas Positives damit verbindet - seien das soziale und berufliche Vorteile, emotionale oder ästhetische Attraktionen oder einfach SprachSammler-Leidenschaften.

$\rightarrow$ Sind die Motivierten willkommen? Wer oder was hat die Demotivierten demotiviert?

$\rightarrow$ Wie kann man die Motivation erhalten und nutzen?

2. Der Einstieg wird leichter, wenn man schon etwas gelernt hat - seien es verschüttete Reste aus der Schulzeit, seien es Wörter und Sätze aus dem Urlaub - alles, was

\footnotetext{
${ }^{13}$ Diese Frage ist sicher eine der Schlüsselfragen bezüglich der Leichtigkeit einer Sprache als Fremdsprache. Soweit ich sehe, gibt es auch dazu keine systematischen Untersuchungen, obwohl ganz offensichtliche sprachspezifische Unterschiede bestehen, die nicht nur von vorgängigen Sprachen des Lerners, sondern auch von anderen Faktoren, z.B. der "Phonetik" abhängen.

14 Achtwöchige Intensivkurse für Studierende der Graduate School am FB Mathematik der TU Kaiserslautern mit jährlich ca. 80 Teilnehmern aus 35-40 Ländern, wobei ein geringer Anteil germanische/romanische Muttersprachen spricht, ein hoher Anteil bereits Englisch gelernt hat.

15 Diese beiden Nennungen gehen wahrscheinlich auf einen impliziten Vergleich mit Englisch zurück. Natürlich spiegeln die Antworten auch hier u.a. Ausrichtungen der Kurse wieder, aber man könnte sicherlich nicht jeden Sprachbereich allein durch spezifische Fokussierungen im Kurs als leicht suggerieren - in die Gegenrichtung ("schwer") wäre das einfacher.
} 
Vertrautheit mit der fremden Sprache herstellt, sie also etwas weniger fremd macht, erleichtert den Einstieg. Dazu gehört auch der vieltausendstündige Phonetik-Kurs fürs Englische, der weltweit auf Radio- und Fernsehsendern läuft.

$\rightarrow$ Was ist weltweit von der Deutschen Sprache bekannt?

$\rightarrow$ Welche Art Vorkenntnisse sind besonders nutzbar bei eventuellem späteren Weiterlernen?

3. Man kann andere, eventuell sogar (nah) verwandte Sprachen. Mehrsprachige Lerner haben schon allein aufgrund ihrer Erfahrungen mit FSE große Vorteile gegenüber einsprachigen. Diese Erfahrungen können durch Kenntnis nahe verwandter Sprachen noch stärker unterstützend wirken. Hierzu sei auf Tertiärsprachenforschung und Untersuchungen zur Mehrsprachigkeit verwiesen. (Hufeisen : 2000) (Bausch : 2004)

4. Gute Lernmöglichkeiten, Kurse, Lernmaterialien und-strategien sowie ein Aufenthalt im Land mit viel Kontakt zu Einheimischen - das galt schon immer als Patentrezept für schnellen FSE. Wenn jeder das weiß, weshalb wird es nicht systematisch umgesetzt? Wieso lernen wir jahrelang jede Woche viele Stunden eine Sprache, um am Ende unbeholfen stotternd das Nötigste zu erledigen, falls wir mal im Land sind, geschweige denn selbstständig angemessene Texte zum realen Gebrauch schreiben.

Was hilft noch?

4.1 Toleranz der Sprecher / Kommunikationspartner gegenüber (Lerner)-Varietäten.

4.2 Die Sprache ist linguistisch sehr gut erforscht und beschrieben.

4.3 Die Forschungsergebnisse werden FSU-praxisrelevant weiterverarbeitet.

4.4 Umfangreiches und differenziertes Angebot an Lehr- und Lernmaterialien.

4.5 Gut ausgebildete Lehrkräfte.

Wie sieht es mit DaF unter diesen Gesichtspunkten aus?

1. Lust auf DaF ist viel häufiger gegeben, als der diesbezüglich verbreitete Pessimismus glauben machen möchte. Die Frage ist, wie lange der Enthusiasmus überlebt und woran er ggf. zerbricht. Bevor also das wachsende Desinteresse an der Wahl von DaF beklagt wird, sollte man sich darum bemühen, bestehendes Interesse zu unterstützen.

$\rightarrow$ Man kann sich also als Lehrer um geeignete Mittel bemühen, Enthusiasmus zu erkennen und für den Lernprozess zu nutzen.

2. Viele Menschen in vielen Ländern können schon ein wenig Deutsch: Das ist im engeren Sinne der Fall, weil es in Schule, Hochschule, Sprachinstitut o.ä. schon gelernt wurde, oder weil man durch Deutschlandaufenthalte schon mit dieser Sprache in Berührung war. Im weiteren Sinne besteht Wissen über Deutschland und Kenntnis von Begriffen/Wörtern durch Internationalismen sowie Export- und Kulturexportgüter. $\rightarrow$ Wenn man als Lehrer und Lehrmaterialproduzent Lerner von der Habenseite her betrachtet, werden plötzlich auch für Anfänger interessante und inhaltlich relevante Kurse möglich. 
3. Eine nah verwandte Sprache - nämlich Englisch - ist die weltweit mit großem Vorsprung verbreitetste Fremdsprache überhaupt, wodurch die meisten erwachsenen Lerner inzwischen schon mindestens eine sehr gute Lernvoraussetzung mitbringen. ${ }^{16}$

$\rightarrow$ Diese Hilfe kann in entsprechenden Gruppen gezielt eingesetzt werden. Ein erster Versuch, hier konkrete Instrumente zu entwickeln liegt mit Marx (im Druck) vor.

4. Grundlagen für gute Lernmöglichkeiten sind für DaF durchaus gegeben.

4.1 Geduld \& Offenheit der L1 Sprecher gegenüber Lernervarietäten mag zum einen daher kommen, dass Deutsch als plurizentrische Sprache schon immer mit Varietäten ${ }^{17}$ lebt und man dies als Bereicherung erlebt (Farbe, Abwechslung, lustig, interessant). Zum andern drückt sich die Neugier auf Fremdes nicht nur in der Reisefreudigkeit, sondern auch in grundsätzlicher Kontaktbereitschaft aus sowie im Interesse an Fremdsprachen.

4.2Deutsch dürfte zu den besterforschten natürlichen Sprachen gehören. Es ist entsprechend viel-beschrieben, vielleicht zuviel-beschrieben: Es gibt keine gemeinsame Beschreibungsgrundlage, so dass schon in einfachen Dingen Verwirrung herrscht - man denke nur an die Bezeichnungen von Wörtern wie deshalb, trotzdem, dazu usw. Vielleicht ist Deutsch auch - im Hinblick auf DaF nicht überall gut beschrieben. Bis heute fehlt nicht nur eine übersichtliche und umfassende Lernergrammatik für Nicht-Philologen, sondern auch eine umfassende und verständliche Beschreibung für DaF-Lehrende.

Zur Frage des Zusammenhanges Schwierigkeit $\leftarrow$ Sprachbeschreibung äußert sich Fries (2001: 642) wie folgt:

"Vielleicht kann die Frage nach dem Schwierigkeitsgrad einer Sprache ... so angegangen werden: Solche Sprachen sind beim Fremdsprachenlernen leichter, welche linguistisch in einer Weise beschrieben sind, dass es Fremdsprachendidaktikern gelingen könnte, das für individuelle Zielsetzungen notwendige sprachliche Wissen in einem akzeptablen Zeitraum zu vermitteln. Im Prinzip sind also Sprachen wie Deutsch, Englisch (...) Chinesisch relativ leicht, weil weitreichend beschrieben und linguistisch expliziert; im Prinzip könnten alle Sprachen leicht sein, wenn da nicht die widrigen Umstände wären, welche die Umsetzung theoretischer Disziplinen in praktische Anwendungen begleiten."

\footnotetext{
16 Beispiele: Sehr viele Lexeme (house, milk, come, brown, under, nineteen ...); systematische Beziehungen (phonetisch $\mathrm{p} \rightarrow \mathrm{f}$ open-offen, ship-Schiff; $\mathrm{d} \rightarrow \mathrm{t}$ day-Tag, do-tun; -ation; ...; morphologisch: sing - sang sung; ...); Kategorisierungen (Einteilung semantischer Bereiche wie Richtung und Bewegung); grammatische Konstruktionen (would/würde + Infinitiv); Adjektivsteigerung (-er -est / good-better-best); diskontinuierliche Konstituenten (wake me up).

${ }^{17}$ Das betrifft nicht nur den Wortschatz und den Klang der Sprache, sondern reicht in Syntax und Morphologie (hat dann gehen müssen - hat müssen dann gehen // hat dort gestanden - ist dort gestanden). Im Gegensatz zur geringen Toleranz, die Lernenden z.B. in Frankreich häufig den Mut zu sprechen nimmt, finden Anfänger in deutschsprachiger Umgebung meist geduldige Kommunikationspartner. Dies gilt im akademischen Milieu für Anglophone leider nicht so, denn sie werden gerne als unfreiwillige Tandempartner zwecks Verbesserung des eigenen Englisch missbraucht.
} 
In dieser Diskrepanz und dem unverhohlenen Zynismus liegen zwei wichtige Gründe für die Ineffizienz von Sprachunterricht: Obwohl etwas klar ist, wird es nicht umgesetzt. In anderen Disziplinen - etwa der Medizin oder den Ingenieurwissenschaften, inzwischen sogar in der Mathematik - gehört die Umsetzung von grundlegenden Erkenntnissen in Praxis ${ }^{18}$ zum wissenschaftlichen Selbstverständnis ${ }^{19}$. In FSE-FSU-bezogenen Bereichen der Sprachwissenschaften dagegen verhindert eine undefinierte aber umso unüberwindlichere Grenze, dass die Forschung, auch die angewandte, intensiv an praktikablen und effizienten Lösungen von alltäglichen Aufgaben in ihrem Bereich arbeitet und sich auch daran messen lassen muss. Es ist aber diese Arbeit von den Lehrern (entsprechend den Ärzten im Bereich Medizin) nicht selbst leistbar.

Der Mangel an Lösungsangeboten für die Praxis verstärkt gegenseitige Vorurteile: Es hilft dem Lehrer in keiner Weise, am Ende von Publikationen mit interessanten Titeln schließlich die Passage mit Praxisbezug zu finden, wenn dort nur vage Hinweise, Desiderata o.ä. stehen. Und es hilft dem einzelnen Wissenschaftler nicht, vor einen riesigen Berg an Forderungen aus der Praxis gestellt zu werden. Aktionsforschung könnte die geeignete Methode für einen Teil dieses Aufgabenberges heißen, gemeinsam vorgehen.

4.3 Nicht nur die Darstellung der Forschungsergebnisse für Endverbraucher im DaFBereich lässt vieles zu wünschen übrig, auch die ausdrückliche Aufgabe, diese Ergebnisse in ganz konkrete Produkte für die Praxis umzusetzen, existiert nicht außerhalb der Lehrerebene. Dass auf diesem Gebiet die größte Veränderung der derzeitigen Praxis notwendig wird, liegt daran, dass nicht nichts existiert, sondern sich vielmehr kontraproduktive Ansätze etabliert haben, die es abzubauen gilt. Hentschel (2002: 107) zeigt z.B., wo überflüssige Regeln in Sprachlehrmaterialien nicht nur nicht helfen, sondern stören:

"Schaden kann eine überflüssige Regel insofern anrichten, als sie den Zugang zum intuitiven Sprachwissen und -verständnis verbaut, indem ein einfach zu lernender Sachverhalt hinter einem Gestrüpp von Regeln und Ausnahmen verschwindet und damit unzugänglich wird."

4.4Ein großes Lehr- u. Lernangebot an DaF-Materialien gibt es in vielfältigen Formen: Kostenlose Kurse der Deutschen Welle, Print-, digitale \& OnlineMaterialien reichlich, Sprachkursangebote aller Art im In- und Ausland. Dabei sind die existierenden Materialien vielzählig aber nicht sehr vielseitig. Denn bis heute dominiert das Lehrbuch alle anderen Materialien, aber es vertritt weitgehend alte Modelle unter immer neuen Oberflächen.

\footnotetext{
18 Beispiel Medizin: Beschreibung von Viren, Krankheitsbildern und -verläufen, schädigenden Einflüssen usw. $\rightarrow$ Medikamente, Therapieformen.

19 Man denke nur an die Selbstverständlichkeit und 'Ehre' der An-Institute an Technischen Hochschulen, wo Praxisrelevanz eine Auszeichnung ist und zum Personal die 'besten Leute' der entsprechenden FB gehören.
} 
4.5 Mit dem Entstehen des Faches DaF zu Beginn der 80er-Jahre sind wir bei der Lehrerausbildung noch in einer jungen Entwicklungsphase, insofern die Mehrzahl der Ausbilder noch aus anderen Fächern - meist Germanistik, Anglistik oder Romanistik - stammt.

Was gute Kurse und Lernmöglichkeiten sind, darüber gibt es viele Ansichten. Es wäre sicher im Vergleich zu aufwändigen Verfahren, mit welchen im Bereich der Sprachlehrforschung gearbeitet wird ${ }^{20}$, nicht schwieriger, einen Gesamterfolg von Sprachlehr/lernmethoden zu überprüfen, indem Erfolge anhand von Kommunikationsfähigkeit in realen Situationen, Wortschatzerwerb, Lesegeschwindigkeit, aufgabengebundener Schreibfähigkeit o.ä. auch nur grob festgestellt würden. Bisher besteht offenbar wenig Interesse an Forschung in dieser Richtung ${ }^{21}$.

Abschließend möchte ich noch drei Kernpunkte betrachten, wie der Zugang zu DaF durch Einrichten guter Lernmöglichkeiten zu erleichtern ist (nähere Ausführungen in Kurtz 2003a, 2003b, 2005).

Der erste zentrale Punkt ist die fokussierte Auswahl von Sprachbereichen. Hierbei stehen vor allem die sog. Routinen für Anfänger im Zentrum, nicht nur für Begrüßung, Abschied und Entschuldigungen, sondern viel umfassender. Der Lehrer kann schon durch seinen eigenen Sprachgebrauch das Einhören in Typisches und Frequentes unterstützen. Mehr noch durch den vermehrten Einsatz von authentischen Texten (Hör-Seh-Lese-Texten). Warum gute Performanz im Bereich ganz oder teilroutinisierten Sprachgebrauchs meist eher argwöhnisch betrachtet wird ("Der kann das nur, weil er es immer so sagt / weil er es sich als fertiges Element abgekuckt hat ..."), ist mir nicht einsichtig. Im ESE ist diese Methode sehr erfolgreich. Auch bei erwachsenen Lernern lässt sich schön das temporäre "Lieblingswort" oder der "Lieblingsausdruck" beobachten, die eine Zeit lang überbenutzt (übergeneralisiert, ausprobiert) werden, um dann ihren angemessenen Platz in der Lernersprache zu finden. Sagen Muttersprachler alles "mit eigenen Worten"?

Rezeptiv beruht die Auswahl hauptsächlich auf Nicht-Auswahl von unnatürlichen PseudoTexten und von tatsächlich zu komplexen Texten, wobei "komplex" nicht grammatisch bedingt ist, sondern vielmehr Texte betrifft, deren Verständnis viel implizites Gruppenwissen $^{22}$ voraussetzt. Bei der Arbeit an Texten kann die Rezeption auf bestimmte Elemente und Eigenheiten fokussiert werden (hier findet die zweite wichtige Auswahl statt), z.B. auf Informationsstruktur, bestimmte Ausdrucksmittel oder semantische Cluster.

Produktiv liegt die Auswahl der Mittel beim Lerner - bestimmte Konstruktionen werden zwar durch den Input nahe gelegt, können aber durch andere Ausdrucksmittel ersetzt werden. Das

\footnotetext{
${ }^{20}$ Introspektionen, qualitative Interviews, quantitative Methoden mit komplexen statistischen Verfahren usw..

${ }^{21}$ Einziges größeres Beispiel mit Zielen in diese Richtung ist das Projekt DIGS (Diehl : 2000); sicher gibt es auch andere solche Projekte, etwa zum Wortschatzerwerb oder zur Messung von Leseleistungen. Die 'große' Forschung allerdings ignoriert den Bereich bisher.

22 Z.B. Wissen über "die 68er Zeit" in Deutschland, über Fernsehserien, Marken, Ereignisse usw., die die Kindheit einer Generation prägten, über lokale Berühmtheiten und sprachliche Eigenheiten, mit denen bestimmte Zeiten oder Personengruppen karikiert werden usw.
} 
Einfordern bestimmter Konstruktionen könnte eine der schärfsten Demotivationswaffen des derzeit üblichen Sprachunterrichts sein. Aufgabe des Lehrers ist eher, Lerner bei der Produktion an die Aufgabe rückzubinden, also immer wieder klar zu machen, ob sie mit ihren Mitteln tatsächlich das Intendierte auch ausdrücken, und falls nein, ihnen Möglichkeiten in ihrer Reichweite anzubieten. Aushandlungsprozesse zur Frage, wie eine Aufgabe angemessen gelöst werden kann, dürfen im Unterricht breiten Raum einnehmen und sind sogar geniale authentische Kommunikationsanlässe. Die Fokussierung liegt hier grundsätzlich auf Bedeutung und Bedeutungsunterschieden und -nuancen.

Der zweite Punkt betrifft ein möglichst vielfältiges, ruhig auch verwirrendes, aber bitte reichhaltiges Angebot an Input, also Hör-, Lese- und Seh-Texten sowie zielsprachliche Gespräche. Je mehr Auswahl Lerner haben, desto eher finden sie erstens Inhalte, die sie zu sprachlicher Anstrengung bewegen, zweitens Ausdrucksmittel, die sich ihnen leicht erschließen und drittens die nötige Abwechslung. ${ }^{23}$ Wenn man die Texte nicht selber mit der Pinzette erstellt, sondern Fundstücke aller Art aus der Umgebung in den Kurs bringt, ist das nicht übermäßig aufwändig. Mit dem Eintritt der Aufgabenvielfalt und -auswahl in den Sprachunterricht kann auch kein permanent zentralisierter Unterrichtsdiskurs stattfinden. Übrigens kann man authentische Texte auch in Auftrag geben, etwa indem man Personen um Beiträge bittet (z.B. Vorstellungen, Erklärungen, Beschreibungen usw.), ggf. mit der Bitte um natürliches Sprechen/Schreiben, ggf. mit der Bitte, sich an Jugendliche oder neu Eingereiste usw. zu wenden; aber gewiss nicht mit Bitten wie "keinen Konjunktiv gebrauchen".

Der dritte Punkt ist Training24: Zum Material gehörige Aufgabenstellungen \& Übungsformen. Vieles vom gängigen Unterrichtsgeschehen ist zwar nicht an sich unnötig, aber im Unterricht deplaziert. Systematische Übung zu Paradigmen, Umformungsaufgaben, Paralleltexterstellung, Bearbeitung von Lückentexten, Abarbeiten von kleinschrittigen Aufgaben und Das_könnte_man_auch_lesen_Texte, all das kann in Selbstlernaktivitäten verlegt werden. Das Kursgeschehen lebt von interaktivem Tun: Hier wird gesprochen und besprochen, geschrieben und überarbeitet und nochmals gegengelesen und wieder überarbeitet, hier wird gesammelt und strukturiert, weniger die Grammatik oder der Wortschatz als vielmehr konkrete Inhalte. ${ }^{25}$ Ein Leitsatz zur Trainerrolle des Lehrers im Anfängerunterricht könnte heißen: Stärken ausbauen lassen, nicht Schwächen bekämpfen ${ }^{26}$. Dazu muss auch ein Wort wie Lernerautonomie ernst genommen werden. Wenn Lerner Dinge tun, die ihnen gelingen, schöpfen sie aus diesem Gelingen Kraft für weitere Betätigung. Ähnlich wie beim ESE muss es eine Inkubationsphase geben dürfen, in welcher ein Sprachbedarf entsteht und Lerner sich einen für sie gangbaren Weg und für sie geeignete Mittel zur Realisierung erobern. Dabei muss durchaus reglementierend (im Sinne der

\footnotetext{
23 Bleyhl betont und begründet diese Notwendigkeit, z.B. in Bleyhl (1997).

24 Training meint hier Sprachunterricht als körperliche Übung, was Bildung nicht ausschließt.

25 Ausarbeitungen zu diesem Punkt demnächst in Kurtz (erscheint).

26 Hierbei geht es nicht um die Förderung von Vermeidungsstrategien, sondern um den Einstieg in die Sprachverarbeitung in der neuen Sprache. Die Aussage bezieht sich weniger auf "Fehlerbereiche" als vielmehr auf Sprachbereiche wie "mündlich-schriftlich" oder "darstellende-diskutierende Texte" bzw. auch Arbeit an Ausdrucksmöglichkeiten mit Fokus auf Erweiterung des Lexikons oder eben von Flexion und Syntax.
} 
Bedeutungsaushandlung) geholfen werden. Den Weg oder die Ausdrucksmittel vorschreiben aber kann höchst destruktiv auf den Erwerbsprozess wirken.

Schluss: Am Anfang der hier gewünschten Veränderungen, also auch der Auffindung leicht zugänglicher Bereiche einer Sprache als Fremdsprache, steht eine geeignete Beschreibung dieser Sprache, die auch geeignet ist, sie in Bezug auf andere Sprachen zu betrachten. Dafür wiederum ist ein geeignetes Beschreibungsinstrumentarium notwendig. Mark Ellison schlägt ein solches vor, das für die Grammatik das leisten kann, was das IPA für die Phonologie leistet. Dabei geht es ihm um die Einheitlichkeit der Beschreibung, nicht um die UG als Glaubensfrage:

"The conclusion that remains is that universality, like the IPA, makes a better convention than fact. It should be used rather than believed." (Ellison : 2000: 524)

Wäre dieses Werkzeug und die damit verbundene Überwindung von Glaubenskriegen in der Linguistik nicht ein enormer Fortschritt - nicht nur für DaF?

Mein Dank gilt Elke Hentschel und den beiden anonymen Gutachtern für ihre wertvollen und lehrreichen Hinweise.

\section{Literaturangaben}

Barkowski, Hans (2001): "Sprachen lernen 'in freier Wildbahn': Zur Bedeutung von Prozeduren der Wahrnehmungsverarbeitung für den Fremdspracherwerb ohne Unterricht". In: Wolff, A. et al. (eds.): Wie schwer ist die deutsche Sprache wirklich? . Regensburg: 555569.

Bauer, Laurie/Trudgill, Peter (eds.) (1998): Language Myths. London.

Bausch, Karl-Richard et al. (eds.) (2004): Mehrsprachigkeit im Fokus. Tübingen.

Bleyhl, Werner (1997): "Fremdsprachenlernen als dynamischer und nichtlinearer Prozess oder: weshalb die Bilanz des traditionellen Unterrichts und auch die der Fremdsprachenforschung 'nicht schmeichelhaft' sein kann". FLuL $26: 219-238$.

Cook, Guy (2003): Applied Linguistics. Oxford.

Demme, Silke (2001): "Transfer und Interferenz: Lernprobleme für DaF-Studierende (nicht nur) mit tschechischer Muttersprache". In: Wolff, A. et al. (eds): Wie schwer ist die deutsche Sprache wirklich? Regensburg: 570-582.

Diehl, Erika (ed.) (2000): Grammatikunterricht: Alles für der Katz? . Tübingen.

Dietrich, Rainer (2003): "Inwiefern kann eine Sprache einfach sein?" LiLi 131: 55-75.

Ellison, Mark T. (2000): "The Universal Constraint Set: Convention, not Fact". In: Dekkers, J. et al. (eds.): Optimality Theory. Oxford: 524-553.

Franceschini, Rita/Klein, Wolfgang (2003): "Einleitung" zu Einfache Sprache $=$ LiLi 131: 5-7.

Fries, Norbert (2001): "Ist Deutsch eine schwere Sprache?". In: Wolff, A. et al. (eds.): Wie schwer ist die deutsche Sprache wirklich? Regensburg: 622-643. 
Hentschel, Elke (2002): "Unnötige Regeln". Linguistik Online 10/2: 101-112. http://www.linguistik-online.de/10_02/hentschel.html.

Hufeisen, Britta (2000): Dritt- und Tertiärsprachenforschung. Flensburg.

Hufeisen, Britta (2001): "Zur Rolle vorher gelernter Fremdsprachen beim Lernen der Fremdsprache Deutsch - Kurzbericht". In: Wolff, A.et al. (eds): Wie schwer ist die deutsche Sprache wirklich? Regensburg: 550 -554.

Klein. Wolfgang (2003): "Warum braucht man eigentlich Flexionsmorphologie?" LiLi 131: 23-54.

Klein, Wolfgang/Dietrich, Rainer (1986): "Simple Language." Interdisciplinary Science Reviews 11/2: 110-117.

Kurtz, Gunde (2003a): "Grammatik im bedeutungsbasierten Sprachenlernen". In: Wolff, A. et al. (eds.): Grammatikvermittlung - Literaturreflexion - Wissenschaftspropädeutik. Regensburg: 557 - 572.

Kurtz, Gunde (2003b): "Lernbeschleunigung - Bedingungen und Schritte zur Realisierung". Zeitschrift für interkulturellen Fremdsprachenunterricht 8/1: 12pp.

Kurtz, Gunde (2005): "DaF-Konzept für Internationale Studiengänge: Integrative Aufgaben in der Hochschule durch objektorientiertes Lernen". In: Motz, M. (ed.): Englisch oder Deutsch in Internationalen Studiengängen. Frankfurt: 153-170.

Kurtz, Gunde (erscheint): "Zielgruppenorientierte Lehr- und Lernmaterialerstellung".

Marx, Nicole (im Druck): Hörverstehensleistungen im Deutschen als Tertiärsprache: zum Nutzen eines Sensibilisierungsunterrichts in 'DaFnE'. (Diss. TU Darmstadt)

Rug, Wolfgang (2001): "Urwälder begehbar machen - Vom richtigen Lehren und Lernen dort, wo Deutsch schwierig ist". In: Wolff, A. et al. (eds.): Wie schwer ist die deutsche Sprache wirklich? Regensburg: 605-621.

Schäfer, Hans-Wilhelm (2002): "Substandardvarietäten des Deutschen als Orientierungshilfen für lernstrategische Wegpunkte". In: Westhof, Gerad/Krumm, Hans-Jürgen (eds): Lehr- und Lernprozesse des Deutschen als Fremdsprache in kognitiver Perspektive. Bern: 127-133.

Serra Borneto, Carlo (2000): "Überlegungen zum Begriff 'Schwierigkeit' - die Lernerperspektive". In: Helbig, B. (ed.): Sprachlehrforschung im Wandel. Tübingen: 339-362.

Serra Borneto, Carlo (2001): "Wie schwer ist Deutsch - Wie ist Deutsch schwer?". In: Wolff, A. et al. (ed.): Wie schwer ist die deutsche Sprache wirklich? Regensburg: 498 - 519.

Wolff, Armin/Winters-Ohle, Elmar (eds.) (2001): Wie schwer ist die deutsche Sprache wirklich? Regensburg.

Wunderlich, Dieter (2003): "Über die Notwendigkeit, Sprachwissenschaft zu betreiben." LiLi $131: 8-22$. 